\title{
Multimodality imaging in cardiology: a statement on behalf of the Task Force on Multimodality Imaging of the European Association of Cardiovascular Imaging
}

\author{
Kevin Fox ${ }^{1 *}$, Stephan Achenbach ${ }^{2}$, Jeroen Bax ${ }^{3}$, Bernard Cosyns ${ }^{4}$, Victoria Delgado ${ }^{3}$, \\ Marc R. Dweck ${ }^{5}$, Thor Edvardsen ${ }^{6}$, Frank Flachskampf ${ }^{7}$, Gilbert Habib ${ }^{8,9}$, \\ Patrizio Lancellotti ${ }^{10,11}$, Denisa Muraru ${ }^{12}$, Danilo Neglia ${ }^{13,14}$, Gianluca Pontone ${ }^{15}$, \\ Ehud Schwammenthal ${ }^{16}$, Udo Sechtem ${ }^{17}$, Mark Westwood ${ }^{18}$, and \\ Bogdan A. Popescu ${ }^{19}$
}

\begin{abstract}
${ }^{1}$ Department of Cardiology, Imperial College Healthcare NHS Trust, Fulham Palace Road, London W6 8RF, UK; ${ }^{2}$ Department of Cardiology, Friedrich-Alexander-University Faculty of Medicine, Krankenhausstraße 12, 91054 Erlangen, Germany; ${ }^{3}$ Department of Cardiology, Leiden University Medical Center, Albinusdreef 22333 ZA Leiden, The Netherlands; ${ }^{4}$ Cardiology Department, CHVZ (Centrum voor Hart en Vaatziekten) - Universtair Ziekhenhuis Brussel, 101 Laarbeklaan, B1090 Brussels, Belgium; ${ }^{5}$ Centre for Cardiovascular Science, University of Edinburgh, Little France Crescent, Edinburgh EH16 4SB, UK; ${ }^{6}$ Oslo University Hospital, Department of Cardiology, Rikshospitalet and University of Oslo Sognsvannsveien 20, 027 Oslo, Norway; ${ }^{7}$ Department of Medical Sciences, Uppsala University, Clinical Physiology and Cardiology, Akademiska, 75185 Uppsala, Sweden; ${ }^{8}$ APHM, La Timone Hospital, Cardiology Department, Rue Saint-Pierre 13385 Marseille Cedex 5, France; ${ }^{9}$ Aix Marseille Univ, IRD, APHM, MEPHI, IHUMéditerranée Infection, 27 Boulevard Jean Moulin 13385 Marseille, CEDEX 5, France; ${ }^{10}$ University of Liège Hospital, Department of Cardiology, GIGA Cardiovascular Sciences, CHU Sart Tilman, 4000 Liège, Belgium; ${ }^{11}$ Gruppo Villa Maria Care and Research, Anthea Hospital, VIA C. ROSALBA, $35 / 3770124$ Bari, Italy; ${ }^{12}$ Department of Cardiac, Thoracic and Vascular Sciences, University of Padua, Via Giustiniani 2, 35128 Padua, Italy; ${ }^{13}$ Fondazione Toscana G. Monasterio (FTGM), Cardiovascular Department, Via Giuseppe Moruzzi, 1, 56124 Pisa, Italy; ${ }^{14}$ Scuola Superiore Sant'Anna, Institute of Life Sciences, Via Santa Cecilia, n. 3 - 56127 Pisa, Italy; ${ }^{15}$ Centro Cardiologico Monzino, IRCCS, Via Carlo Parea 4, Milan 20138, Italy; ${ }^{16} \mathrm{Tel}$ Aviv University and Heart Center Sheba Medical Center, Emek Doran St 52621 Ramat Gan, Israel; ${ }^{17}$ Department of Cardiology, Robert Bosch Krakenhaus, Auerbachstraße 110, 70376 Stuttgart, Germany; ${ }^{18}$ Barts Heart Centre, St Bartholomew's Hospital, West Smithfield, London EC1A 7BE, UK; and ${ }^{19}$ Department of Cardiology, University of Medicine and Pharmacy “Carol Davila” - Euroecolab, Emergency Institute of Cardiovascular Diseases "Prof. Dr. C. C. Iliescu”, Sos. Fundeni 258, Sector 2, 022328 Bucharest, Romania
\end{abstract}

Received 15 June 2018; revised 21 August 2018; editorial decision 11 September 2018; accepted 4 October 2018

\section{Introduction}

Imaging of the heart and related structures has evolved over the last 100 years progressively introducing different modalities. The initial use of X-ray was enhanced by echocardiography and now complemented by cardiac magnetic resonance (CMR), cardiac computed tomography (CT), nuclear cardiology (single-photon emission tomography and positron emission tomography, PET) together with fusion modalities. Their integration has until recently been based as much on the timeline of their arrival as a clinical tool, as on their clinical utility. In the 21 st century, it is essential we use and integrate these different modalities to the best advantage of our patients ${ }^{1-4}$ (Table 1).

The European Association of Cardiovascular Imaging (EACVI) established a Task Force on Multimodality Imaging (TFMMI).The aim was to identify a consensus on the progression of multimodality imaging and especially the role of the EACVI in developing, promoting and implementing multimodality imaging to improve patient care.
This was in accordance with the mission statement of the European Association of Cardiovascular Imaging ${ }^{5}$ as well as aligning with the European Society of Cardiology (ESC) strategic plan for 2016-2020.

There have been previous publications from Europe and North America on training and competence in different imaging modalities, ${ }^{7-}$ ${ }^{13}$ but these have not discussed in detail specifics of training in multimodality imaging (MMI) and the challenges involved in progressing a multimodality approach. We aim to set out core principles in this statement with a view to on going work and publications on specific areas such as training and image modality selection for different clinical scenarios.

\section{Process}

The Task Force was established in August 2017 to represent key expertise and opinion leaders across cardiovascular imaging. The Task

\footnotetext{
* Corresponding author. Tel: +44 20331 38984, Fax: +44 208331 18182, Email: k.fox@imperial.ac.uk

Published on behalf of the European Society of Cardiology. All rights reserved. @ The Author(s) 2018. For permissions, please email: journals.permissions@oup.com.
} 
Table I Cardiac imaging modalities

\begin{tabular}{|c|c|c|c|}
\hline Modality & Underlying principle & Primary attributes & Key uses \\
\hline Echocardiography & $\begin{array}{c}\text { Analysis of reflected ultrasound } \\
\text { waves at tissue interfaces }\end{array}$ & $\begin{array}{l}\text { High frame rate, high spatial reso- } \\
\text { lution, very high temporal poten- } \\
\text { tially (e.g. Doppler) bedside, safe }\end{array}$ & $\begin{array}{l}\text { Cardiac structure and function. Valve } \\
\text { structure and function. Identification } \\
\text { of ischaemia and viability (stress } \\
\text { echo) }\end{array}$ \\
\hline Magnetic resonance imaging & $\begin{array}{l}\text { Electromagnetic waves emitted } \\
\text { from protons in response to a } \\
\text { pulsatile magnetic field }\end{array}$ & $\begin{array}{l}\text { Tissue characterization Spatial reso- } \\
\text { lution. Safety }\end{array}$ & $\begin{array}{l}\text { Cardiac muscle and chamber structure } \\
\text { function; tissue characterization, is- } \\
\text { chaemia (metabolism) }\end{array}$ \\
\hline $\mathrm{CT}$ imaging & Differential absorption of X-rays & $\begin{array}{l}\text { Cardiac structure including coronary } \\
\text { arteries; high spatial resolution }\end{array}$ & $\begin{array}{l}\text { Cardiac including valve structure and } \\
\text { coronary circulation. FFR }\end{array}$ \\
\hline $\begin{array}{l}\text { Nuclear Imaging (including } \\
\text { combined with CT/CMR) }\end{array}$ & $\begin{array}{l}\text { Uptake and wash out of radio } \\
\text { tracers (gamma emitting or } \\
\text { positron emitting) }\end{array}$ & $\begin{array}{l}\text { Functional assessment of myocardial } \\
\text { perfusion, metabolism, innervation } \\
\text { and vascular inflammation. Possible } \\
\text { quantitation and integration }\end{array}$ & $\begin{array}{l}\text { Detection of myocardial ischaemia, via- } \\
\text { bility, and denervation. Detection of } \\
\text { infection }\end{array}$ \\
\hline
\end{tabular}

Force undertook initial surveys and conference calls and then, face to face meetings in December 2017. Further conference calls, surveys and questionnaires took place during the drafting of the statement. External expertise was utilized as needed.

\section{Definitions}

A definition of $\mathrm{MMI}$ was agreed to provide a framework for the project.

Imaging of the heart based around the patient, and their disease pathology and presentation, utilizing information from one or more imaging modalities performed separately or in combination and offering the most clinically effective and cost effective testing for the patient.

In terms of knowledge and skill, different modalities use the terms Level 1, Level 2, and Level 3 in different ways. For example, Level 1 in cardiac CT provides a basic understanding of the technique, while Level 1 in echocardiography implies an ability to undertake limited scanning. We use in this document a classification based around the terminology in the ESC Core Curriculum for the General Cardiologist (Table 2). ${ }^{14}$

\section{Organization of cardiovascular imaging services}

Teams of individuals work most effectively when geographically colocated. Therefore, the ideal configuration would be the co-location of all the modalities. However, this is impractical if not impossible in existing facilities. Never the less its consideration should be included in the design process for cardiovascular facilities. The benefits of colocation must be balanced with other factors including patient flows and construction requirements. Where hospitals have access to limited modalities they should form networks to ensure equity of access for their patients to the full range of cardiac imaging.

If facilities cannot be co-located then the co-location of staff who report imaging investigations should be prioritized.
This makes a difference to:

- Imaging specialists who can collaborate and discuss and draw on different expertise (e.g. by co-locating radiologists and cardiologists).

- Non-imaging cardiologists who can come to one place to get best advice on how to image their patients. This allows the integration of the patient's clinical scenario and their wishes with proportionate and cost effective use of imaging (Table 3).

This co-operation is not just about location. It is also about the working environment. Having workstations fully capable of accessing and manipulating outputs from all the imaging hardware in standardized formats contributes to an integrated multimodalitiy diagnostic approach and breaking down silos within hospitals. Seamless integration between electronic patient records and imaging software should be the aim. There are commercial and cost considerations in accessing multiple imaging modalities on single workstations but easy access and integration is clinically advantageous and should be progressed within hospitals and by manufacturers.

Whether imaging specialists are co-located or not, it is essential that regular clinical meetings bring together multimodality expertise, together with other clinicians, as part of the multidisciplinary team approach to patient care (Take home figure).

Unimodality meetings still have a role to deal with specific issues such as training and quality assurance.

The EACVI should provide a strong MMI focus to its educational activities including a dedicated modular MMI teaching programme and the EACVI congress. However, there is still a need for unimodality meetings, textbooks, webinars, and other educational materials. In 2020, the EACVI will support a single 'EACVI Congress' with a focus on $\mathrm{MMI}^{15}$

\section{Key statement}

Co-location of cardiovascular imaging is recommended, particularly bringing together imaging expertise for shared reporting and decision making. 


\section{Table 2 Levels of competence}

Level 1 Experience of selecting the appropriate diagnostic or therapeutic modality and interpreting results or choosing an appropriate treatment for which the patient should be referred. This level of competency does not include performing a technique, but participation in procedures during training may be valuable (for echo we include basic image acquisition as a Level 1 skill)

Level 2 Level II goes beyond Level I. In addition to Level I requirements, the trainee should acquire practical experience but not as an independent operator. They should have assisted in or performed a particular technique or procedure under the guidance of a trainer. This level also applies to circumstances in which the trainee needs to acquire the skills to perform the technique independently, but only for routine indications in uncomplicated cases

Level 3 Level III goes beyond the requirements for Levels I and II. The trainee must be able independently to recognize the indication, perform the technique or procedure, interpret the data, and manage the complications

\section{Table 3 Case scenario}

\begin{tabular}{ll}
\hline Clinical problem & A 47-year-old man presents with 3 weeks of fever having undergone mech- \\
anical aortic valve placement 3 years previously \\
ECG shows a PR interval of $218 \mathrm{~ms}$ \\
Blood cultures do not show any bacterial growth \\
He is discussed with cardiac imaging and radiology experts \\
Mild likely paraprosthetic regurgitation is seen but images are degraded by \\
Transthoracic \\
echocardiography
\end{tabular}

echocardiography

artefact from the leaflets

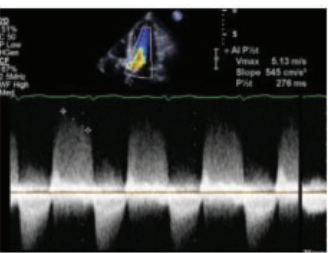

Transoesophageal

Moderate paraprosthetic regurgitation localized to the posterior annulus echocardiography

PET-CT

Increased activity along the posterior annulus

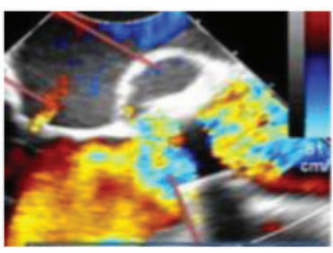
CT imaging and coronary
angiography
Cavity adjacent to the aortic prosthesis

Unobstructed coronary arteries
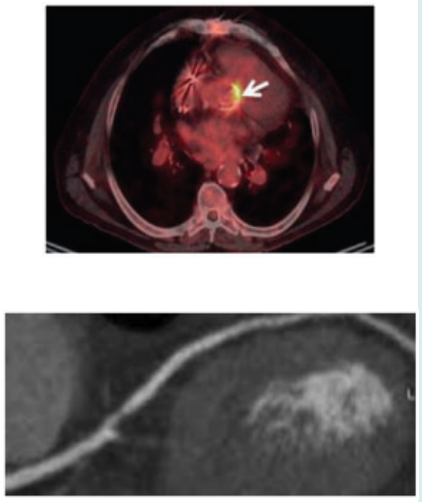

Patient management

Following MDT discussion the patient is treated with 6 weeks of antibiotics and then undergoes redo aortic valve replacement

\section{Current workforce}

At present cardiovascular imaging is primarily provided by specialists with backgrounds in cardiology, nuclear medicine, and radiology.
This is likely to continue at least in the short and medium term. Therefore, their integration and collaboration should be a priority within institutions. There are clear advantages to this collaboration as cardiologists, radiologists, and nuclear medicine experts may provide 


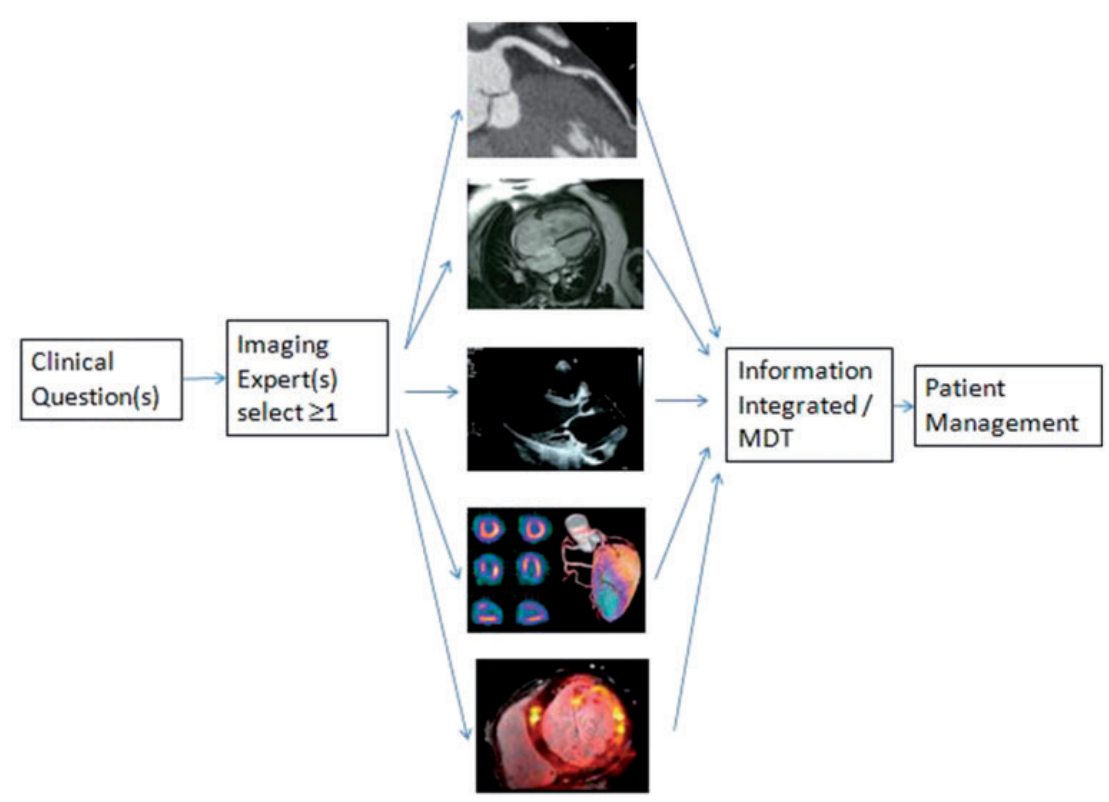

Take home figure Multimodality imaging in clinical practice.

complementary inputs through different backgrounds in training and not fully overlapping skill sets. Non-cardiologists can provide unique expertise in diagnosing clinically important thoracic abnormalities outside the heart when CT, CMR, and PET are performed.

The use of echocardiography on wards, in emergency rooms and in cardiac catheter laboratories has largely become the role of cardiologists and this will continue. However, basic echocardiographic assessment, for example using $\mathrm{FEEL}^{16}$ and $\mathrm{FAST}^{17}$ protocols or focus cardiac ultrasound ${ }^{18}$ are expanding the skill of cardiac ultrasound to other clinicians.

The current workforce contains many specialists with single modality backgrounds. These individuals should be strongly encouraged to obtain a basic understanding of additional modalities, and opportunities promoted to facilitate their ability to perform and interpret images in additional modalities (Level 2). The EACVI should be facilitating training through educational opportunities on-line and at its meetings and by provision of grants, and exchanges.

Currently, allied health care professionals (physiologists, technicians, and radiographers) tend to support single modalities. They should also be encouraged and supported to gain some understanding of multiple modalities.

\section{Key statement}

All imaging specialists with expertise in one modality should be encouraged and facilitated to achieve a basic capability to perform and report in one or more additional modalities.

\section{Training}

Future trainees in the specialty of cardiology and those subspecializing in imaging should be trained in a MMI environment. They should be encouraged to think about the patient and clinical problem and relate the choice of cardiac imaging modality/modalities to those needs.

Imaging principles and physics should be taught holistically rather than as modality silos.

This means that training programmes within hospitals currently only able to offer a limited range of imaging modalities, must form collaborations to provide comprehensive training.

Trainees are likely to have some service and on-call role and therefore within an overall MMI framework an early priority should be the acquisition of echocardiography skills. Early in their general cardiology training, they should achieve a basic understanding of all modalities (Level 1) but additionally attain competence to perform and report echocardiograms particularly as it relates to acute cardiac presentations (Level 2).

Subsequently, all trainees should achieve basic competence in performing and reporting echocardiograms across the full spectrum of pathologies (Level 3).

Trainees not specializing in imaging may well need to develop Level 3 practical performance and reporting expertise in modalities appropriate for their sub-specialization.

Trainees choosing to sub-specialize in cardiac imaging should achieve as a minimum a basic competence in performing and reporting all modalities (Level 2). The further standard should be completion of training in at least two modalities (Level 3). In fact the degree of expertise should be at least to the point where they can act as a senior contributor to the service work of both modalities (increasingly a requirement for clinical jobs in cardiac imaging) and provide leadership and training. There may be exceptions for example where a trainee wishes to pursue an academic career focused on a narrow area of cardiac imaging. However, while some individuals will focus on a single modality to achieve expertise to provide international leadership in research and practice, others will be able to produce high quality research utilising more than one modality. 


\section{Key statements}

Trainees should achieve basic understanding in all modalities and practical capability to perform and report echocardiograms early in their general cardiac training.

Trainees subspecializing in imaging should achieve practical capability to perform and report simple studies in all modalities and in almost all cases expertise in two or more modalities by the end of their training.

\section{Challenges to developing a multimodality imaging approach and how to overcome them}

We are proposing a call to action for hospitals and individuals. We understand:

(1) Not all hospitals across Europe have access to all imaging modalities. This affects the clinical service as well as training.

(2) Current architectural layouts do not facilitate collaboration.

(3) Institutions are working within restricted budgets.

(4) 'Silos' are common in hospitals and represent significant barriers to collaboration.

Therefore, we acknowledge that some of the recommendations are aspirational and no specific timelines have been set in this document.

There are further issues that act as barriers to a multimodality imaging service:

(A) Management and financial flows: budgets and financial flows influence practice. Currently, most hospitals have separate managerial structures and budgets for cardiology, radiology, nuclear medicine, and even between cardiac imaging modalities. With limited resources this encourages competition rather than collaboration. In many situations volume is currently an important financial driver rather than appropriateness. However, it is likely that the most clinically effective imaging of a patient will also be the most cost effective.

Hospitals should develop managerial structures and financial flows that encourage collaboration between modalities. At its simplest this may be appointing a clinical and managerial lead with a complete oversight for cardiovascular imaging which would encourage balanced investment in imaging facilities.

(B) Challenges to individuals: in transforming cardiac imaging services personal anxieties must be identified and overcome. Change can be challenging and even threatening. Single modality cardiac imaging specialists will need to broaden their knowledge and skills. Imaging departments will also need to adapt. Time and effort are needed to build trust which is essential to facilitate change.

(C) Non-imaging cardiologists: these equally need to embrace the multimodality approach. They need access to education and the flexibility to adapt their imaging requests to the current most appropriate modality or modalities for their clinical questions. Local arrangements will need to be established around altering test requests. This will be facilitated by the co-location of the cardiac imaging team.

(D) National regulations: International and National regulations cover aspects of cardiac imaging especially regarding radiation exposure and use of radio-isotopes. These have to be incorporated in the design of departments and staff training.

\section{Key statement}

Successfully creating a multimodality approach to cardiac imaging requires collaboration and overcoming managerial, financial, personal, and regulatory barriers.

\section{Research}

The underpinning principle of this document is that a multimodality approach to cardiac imaging improves patient care and is cost effective. There is limited confirmatory evidence to support this statement. While research is needed to continue to develop all aspects of cardiac imaging, studies are needed specifically to confirm the clinical and financial benefits of a multimodality approach. Within research projects themselves it is important the optimal modality or modalities are identified to answer the scientific question.

\section{What is the role of the EACVI?}

The mission statement of the EACVI is:

We promote excellence in clinical diagnosis, research, technical development, and education in cardiovascular imaging

The EACVI must use its leadership role to be active in progressing a multimodality approach.

The EACVI should:

(1) Have a MMI focus to its annual meeting, for example, through the changes already planned from 2020 with a revamped EACVI congress.

(2) Facilitate research through providing forums for collaboration and considering using its resources (grants) to advance research.

(3) Offer education to single modality imaging specialists, e.g. 'CMR for echo cardiologists'.

(4) Facilitate exchanges for education.

(5) Provide a strong MMI focus to its educational activities including a dedicated modular MMI teaching programme.

(6) Support a MMl approach to training through different means (e.g. text books, on line materials, webinars, conferences).

(7) Develop a curriculum for trainees that is based on a multimodality approach (topics such as strengths and weaknesses of modalities and selecting the correct modality/modalities).

(8) Demonstrate a commitment to $\mathrm{MMl}$ cardiac imaging through its certification processes. Actions may include the curricula for unimodality certifications including the requirement for a basic understanding of the relative role of other modalities, and consideration of a 'Certification in Multimodality Imaging'.

(9) Use the influence, expertise, and resources of the EACVI to provide education and to overcome local, national and international challenges to progressing MMI.

(10) Continue the work of this Task Force by producing further detailed statements on MMI curricula, training, clinical utility of $\mathrm{MMI}$ in different patient scenarios and quality assurance.

The European Heart Journal Cardiovascular Imaging is the journal of the EACVI. It already provides an important forum for multimodality studies and debate. Without impinging on its editorial independence the EACVI should be further encouraging the acceptance of high quality articles on the clinical and cost effectiveness of multimodality imaging, including testing imaging pathways for different clinical presentations. 


\section{Summary}

Best clinical care will need an appropriate use of one or more imaging modalities. The EACVI is committed to enhancing through education and research the multimodality approach.

\section{Acknowledgements}

We thank the following colleagues for their helpful comments used in preparation of this statement: Marcelo Di Carli, Bas Bekkers, Matteo Cameli, Nuno Cardim, Erwan Donal, Joost van Melle, Natalia Maroz-Vadalazhskaya, Leopoldo Perez de Isla, Francisco Sampaio.

Conflict of interest: none declared.

\section{References}

1. Lancellotti P, Nkomo VT, Badano LP, Bergler-Klein J, Bogaert J, Davin L, Cosyns B, Coucke P, Dulgheru R, Edvardsen T, Gaemperli M, Galderisi M, Griffin B, Heidenreich P, Nieman K, Plana J, Port S, Scherrer-Crosbie M, Schwartz R, Sebag I, Voight J-U, Wann S, Yang P. Expert consensus for multi-modality imaging evaluation of cardiovascular complications of radiotherapy in adults: a report from the European Association of Cardiovascular Imaging and the American Society of Echocardiography. Eur Heart J Cardiovasc Imaging 2013;14:721-740.

2. Habib G, Bucciarelli-Ducci C, Caforio ALP, Cardim N, Charron P, Cosyns B, Dehaene A, Derumeaux G, Donal E, Dweck MR, Edvardsen T, Erba PA, Ernande L, Gaemperli O, Galderisi M, Grapsa J, Jacquier A, Klingel K, Lancellotti P, Neglia D, Pepe A, Perrone-Filardi P, Petersen SE, Plein S, Popescu BA, Reant P, Sade LE, Salaun E, Slart RHJA, Tribouilloy C, Zamorano J, Delgado V, Haugaa K, Vijayaraghavan G; EACVI Scientific Documents Committee; Indian Academy of Echocardiography. Multimodality imaging in restrictive cardiomyopathies: an EACVI expert consensus document in collaboration with the "Working Group on myocardial and pericardial diseases" of the European Society of Cardiology. Eur Heart J Cardiovasc Imaging 2017;18:1090-1121.

3. Haugaa KH, Basso C, Badano LP, Bucciarelli-Ducci C, Cardim N, Gaemperli O, Galderisi M, Habib G, Knuuti J, Lancellotti P, McKenna W, Neglia D, Popescu BA, Edvardsen T, Delgado V, Cosyns B, Donal E, Lombardi M, Muraru D, Kauffmann P, Jurcut R, Klein JB, Sade LE; EACVI Scientific Documents Committee, EACVI Board members and external reviewers. Comprehensive multi-modality imaging approach in arrhythmogenic cardiomyopathy-an expert consensus document of the European Association of Cardiovascular Imaging. Eur Heart J Cardiovasc Imaging 2017;18:237-253.

4. Cardim N, Galderisi M, Edvardsen T, Plein S, Popescu BA, D’Andrea A, Bruder O, Cosyns B, Davin L, Donal E, Freitas A, Habib G, Kitsiou A, Petersen S, Schroeder S, Lancellotti P. Role of multimodality cardiac imaging in the management of patients with hypertrophic cardiomyopathy: an expert consensus of the European Association of Cardiovascular Imaging. Eur Heart J Cardiovasc Imaging 2015;16:280.
5. EACVI strategy. https://www.escardio.org/Sub-specialty-communities/EuropeanAssociation-of-Cardiovascular-Imaging-(EACVI)? hit=metanav (28 April 2018).

6. ESC strategic plan. https://www.escardio.org/static_file/Escardio/Web/About/ Documents/ESC-Strategic-plan-2016-2020.pdf (28 April 2018).

7. Popescu BA, Andrade MJ, Badano LP, Fox KF, Flachskampf FA, Lancellotti P, Varga A, Sicari R, Evangelista A, Nihoyannopoulos P, Zamorano JL, Derumeaux G, Kasprzak JD, Roelandt JR. European Association of Echocardiography recommendations for training, competence, and quality improvement in echocardiography. Eur J Echocardiogr 2009;10:893-905.

8. Ryan T, Berlacher K, Lindner JR, Mankad SV, Rose GA, Wang A. COCATS 4 Task Force 5: training in-echocardiography. J Am Coll Cardiol 2015;65: 1786.

9. Petersen SE, Almeida AG, Alpendurada F, Boubertakh R, Bucciarelli-Ducci C, Cosyns B, Greil G, Karamitsos T, Lancellotti P, Stefanidis A, Tann O, Westwood M, Plein S; Education Committee of European Association of Cardiovascular Imaging Association (EACVI). Update of the European Association of Cardiovascular Imaging (EACVI) Core Syllabus for the European Cardiovascular Magnetic Resonance Certification Exam. Eur Heart J Cardiovasc Imaging 2014;15: 728-729.

10. Kramer CM, Hundley WG, Kwong RY, Martinez MW, Raman SV, Ward RP. COCATS 4 Task Force 8: training in cardiovascular magnetic-resonance-imaging. J Am Coll Cardiol 2015;65:1822.

11. Pontone G, Moharem-Elgamal S, Maurovich-Horvat P, Gaemperli O, Pugliese F, Document Reviewers Westwood M, Stefanidis A, Fox K, Popescu B. Training in cardiac computed tomography: EACVI certification process. Eur Heart J Cardiovasc Imaging 2018;19:123-126.

12. Narula J, Chandrashekhar YS, Dilsizian V, Garcia MJ, Kramer CM, Malik S, Ryan T, Sen S, Wu J. COCATS 4 Task Force 4: training in multimodality imaging. J Am Coll Cardiol 2015;65:1778.

13. Douglas PS, Cerqueira MD, Berman DS, Chinnaiyan K, Cohen MS, Lundbye JB, Patel RAG, Sengupta PP, Soman P, Weissman NJ, Wong TC, Asch F, Bateman TM, Berman DS, Biesbrock G, Brinker J, Brophy D, Cerqueira M, Chinnaiyan K, Cohen M, Dilsizian V, Douglas PS, Dudley J, Epstein F, Gillam L, Lundbye J, McCallister B, Narula J, Patel RAG, Reynolds D, Schoepf UJ, Sengupta PP, Shah N, Soman P, Udelson JE, Valeti US, Walsh MN, Ward RP, Weigold WG, Weissman NJ, White RD, Wolinsky D, Wong TC, Zoghbi WA. The future of cardiac imaging. JACC Cardiovasc Imaging 2016;9:1211.

14. Gillebert TC, Brooks N, Fontes-Carvalho R, Fras Z, Gueret P, Lopez-Sendon J, Salvador MJ, van den Brink RBA, Smiseth OA, Griebenow R. ESC Core Curriculum for the General Cardiologist (2013). Eur Heart J 2013;34: 2381-2411.

15. Popescu BA. The EACVI. Eur Heart / 2018;39:1582-1584.

16. FEEL. FEEL. https://www.escardio.org/static_file/Escardio/Subspecialty/EACVI/pos ition-papers/echo-in-emergency-full.pdf (29 April 2018).

17. FAST. FAST. http://www.trauma.org/archive/radiology/FASTfast.htm (28 April 2018).

18. Neskovic AN, Skinner H, Price S, Via G, De Hert S, Stankovic I, Galderisi M, Donal E, Muraru D, Sloth E, Gargani L, Cardim N, Stefanidis A, Cameli M, Habib G, Cosyns B, Lancellotti P, Edvardsen T, Popescu BA. Focus cardiac ultrasound core curriculum and core syllabus of the European Association of Cardiovascular Imaging. Eur Heart / Cardiovasc Imaging 2018;19:475-481. 